\title{
Treatment of Inflammatory Gingival Lesion Caused by Vertical Root Fracture
}

\author{
Hamid Moghaddas and Arezou Pezeshkfar \\ Department of Periodontics, Faculty of Dentistry, \\ Shahid Beheshti University of Medical Sciences, Tehran, Iran
}

\begin{abstract}
Treatment of a tooth with vertical root fracture can be difficult and unpredictable. Quite often, the most predictable treatment possible is simply extraction. This study presents, a patient with an inflammatory gingival lesion on the labial aspect of the maxillary right central incisor due to vertical root fracture. This diagnosis confirmed during surgery. There was also a super contact on the mentioned tooth. Root canal therapy was done and super contact eliminated. After 5 weeks, the gingival gained normal appearance. A total of 12 months follow up showed that the results remained stable and the tooth treated remained asymptomatic. It is necessary to emphasize that it is not possible to extend the result of this treatment to all patients with vertical root fracture and at the present there is no predictable treatment for such teeth and extraction may be the only choice of treatment.
\end{abstract}

Key words: Vertical root fracture, cracked tooth, inflammatory gingival lesion, internal root resorption, predictable, eliminated

\section{INTRODUCTION}

Unfortunately, the incidence of cracks in teeth seems to be increasing. People are living longer and keeping their teeth longer. As a result, patients are more likely to have complex restorative and endodontic procedures that remove tooth structure, leaving teeth more susceptible to cracks.

People of all ages who are living in more stressful condition be prone to crack-inducing habits such as bruxism and clenching. One factor that contributes to confusion surrounding the issue of cracked teeth is that various researchers have suggested a number of inconsistent terms to describe tooth cracks. Because the location, direction and extent of a crack have a profound effect on the choice of treatment which is important to be clarified.

In 1997, the American Association of Endodontists, published an study in this issue and rendered the five types of tooth cracks as follows:

- Craze line affect only the enamel

- Fracture cusp

- Cracked tooth

- Split tooth begin on the occlusal surface and extent apically affecting enamel, dentin and possibly the pulp (Fig. 1a-c)
- Vertical Root Fracture (VRF) begins in the root usually on the buccal-lingual plane. A VRF may extent the length of the root or occurs as a shorter crack at any level along the root

The crack may or may not extent to both buccal and lingual surfaces (Fig. 1d). Because VRFs present minimal sign and symptom, they usually go unnoticed until periradicular pathosis occurs. Then, they are very difficult

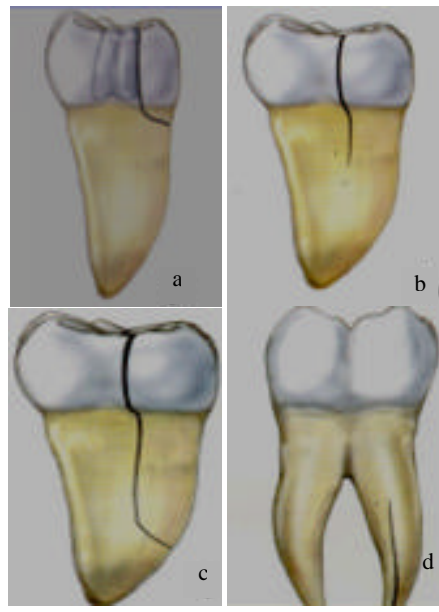

Fig. 1: (a) Fractured cusp; b) Cracked tooth; c) Split tooth and d) Vertical root frature

Corresponding Author: Hamid Moghaddas, Department of Periodontics, Faculty of Dentistry, 
to diagnose because they mimic other conditions. The recommended treatment is almost exclusively extraction or removal of the cracked root therefore, care must be taken to avoid incorrect diagnosis (Andreasen et al., 1991; Bender and Freedland, 1983a, b; Blaser et al., 1983; Brown et al., 1972; Burke, 1992; Cameron, 1964; Cameron, 1976; Carter et al., 1983; Caufield, 1981; Cavel et al., 1985; Christensen, 1993; Dang and Walton, 1989; Eakle et al., 1986; Ehrmann and Tyas, 1990; Feiglin, 1995; Gher et al., 1987; Gibbs, 1954; Glass and Zander, 1949; Gutmann and Rakusin, 1994; Gutmann and Harrison, 1991; Gutmann and Gutmann, 1995; Harper et al., 1980; Harrington, 1979; Harvey et al., 1981; Hiatt, 1973; Holcomb et al., 1987; Howe and McKendry, 1990; Huang et al., 1992; Johnson et al., 1976; Josell, 1995). Because VRFs mimic periodontal disease or failed root canal treatment, these cases often result in refer to a periodontist or endodontist for evaluatation. Researchers are looking into new treatments but as yet no method of saving the cracked root has proven practical or effective in long term.

Case report: The patient was a 21 years old woman attending the Shahid Beheshti Dental School (Tehran, Iran) with the chief complaint of presense of an inflammatory lesion on the facial gingival of the right central incisor (Fig. 2a). She had no systemic disorder and was not taking any medication. Orthodontic therapy had been done for the patient about 8 years ago by a general dentist but relapse occurred. About 2 years ago, she underwent orthodontic therapy again and few months after finishing it, the lesion appeared on the gingiva and increased in size. About 1 year ago, the lesion was eradicated by cauterization. But complete healing did not achieve and the lesion reappeared. The patient did not remember any history of trauma to the anterior teeth. She was healthy periodontally, probing depth was normal in all sites in the mouth except the right central incisor which had $8 \mathrm{~mm}$ pocket on the labial aspect but its mobility was in normal range. Radiographic evaluation showed signs of internal resorption (Fig. 2b). X-ray examination of the tooth from different directions led us to conclude that the cause of radiolucency is an internal root resorption. The tooth was not sensitive to percussion but it did not respond to electric pulp tester and cold tests. The patient had not any complaint about the mentioned tooth except esthetic problem because of her high lip line during smiling, the lesion became apparent therefore, she was seeking for the treatment. At first, attempt was made to do root canal therapy. After pulpectomy of the tooth, calcium hydroxide was inserted in the root canal. After 2 weeks, surgery was done at the labial aspect of the tooth with two purposes:

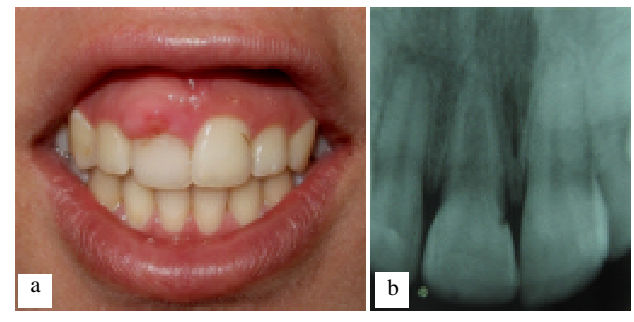

Fig. 2: (a) Clinical view and b) radiographic view before treatment

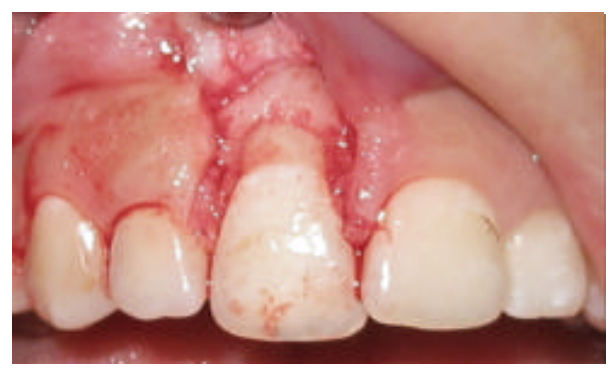

Fig. 3: Design of incision and elimination of lesion

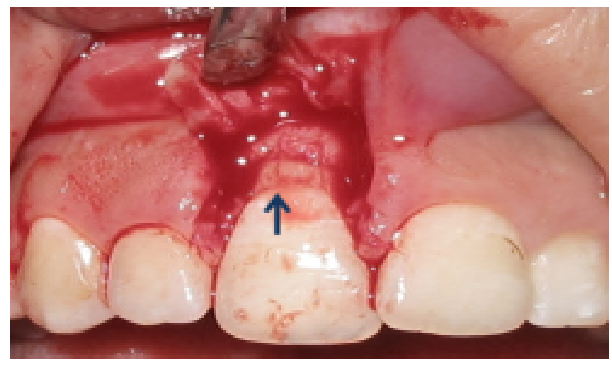

Fig. 4: Visibilty of the crack on the root surface

- Elimination of the gingival lesion

- Evaluation of the root surface and seeking the cause of the lesion

Surgery procedure: After administration of local anesthesia, the lesion was eliminated by a horizontal incision. Thereafter, two vertical incisions were made at the mesial and distal line angles of the tooth \# 8 and a full thickness flap was reflected (Fig. 3 and 4). Then horizontal bone loss was appeared at the labial aspect of the tooth. In addition, there was a fine crack on the root surface. The crack began from the CEJ and extended to the alveolar crest. The crack was too fine to observe but by adding a droplet of adjacent blood to the area, it was seen better. Based on the classification that described previous, the crack was diagnosed as a vertical root fracture. This tooth has a premature contact on protrusive movement (Fig. 5). Therefore, occlusal adjustment was accomplished (Fig. 6). 


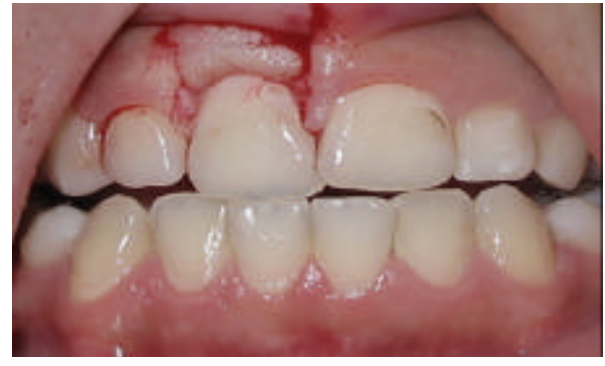

Fig. 5: Super contact in protrusive movement

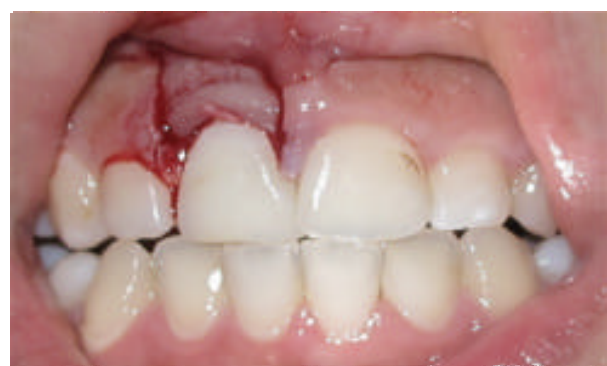

Fig. 6: Ocdusal adjustment

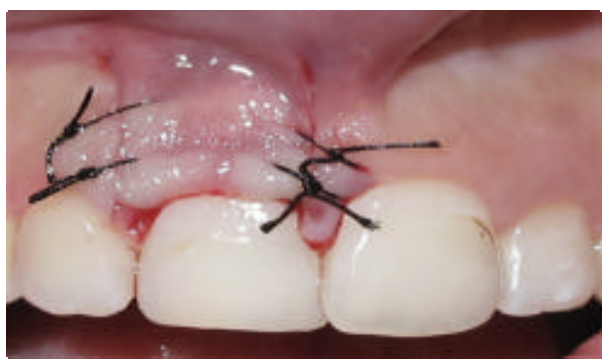

Fig. 7: Flap positioned coronally and stabilized by sutures

Then, root surface was planned by hand instruments and conditioned by mixture of doxycycline and normal saline for $1 \mathrm{~min}$. Thereafter with a periosteal incision, the flap positioned coronally for coverage, the denuded root and stabilized by interrupted sutures (Fig. 7 and 8). The area was covered by periodontal dressing. The patient was instructed to take analgesic medication (ibuprofen $400 \mathrm{mg}$ t.i.d.) as necessary and rinse with chlorhexidine $(0.2 \%)$ twice daily for 3 weeks.

It was recommended not to perform mechanical oral hygiene for at least 3 weeks. Soft diet regime was suggested to the patient. Dressing and sutures were removed at 1 week and the area was irrigated by chlorhexidine. After 2 weeks, the root canal therapy was completed, apical portion of the canal was obturated with gutta percha by lateral condensation technique and other

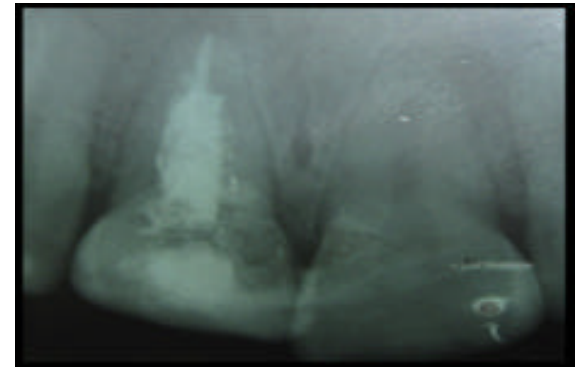

Fig. 8: Radigraphic view after treatment
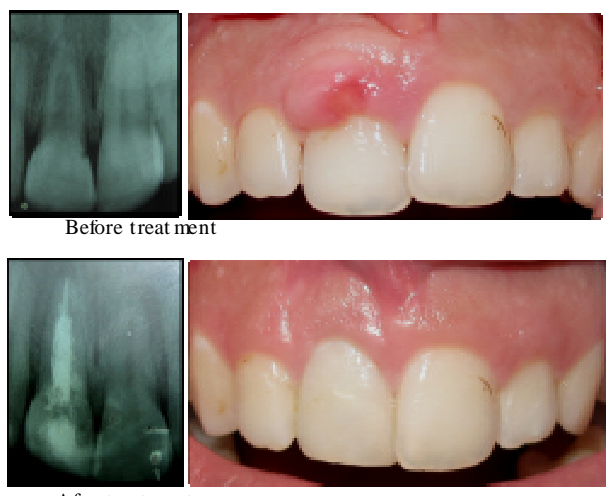

After treat ment

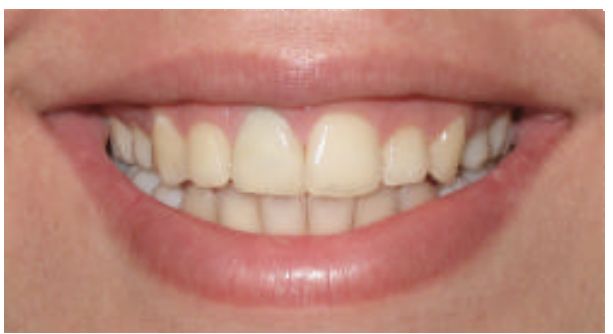

Frontal view of the pat ient 12 months after treatment

Fig. 9: Clinical view 5 weeks after treatment

portions by warm gutta percha. After 5 weeks, the gingival had normal appearance and stippling was apparent (Fig. 9).

\section{DISCUSSION}

Treatment of a tooth with vertical root fracture can be difficult and unpredictable. Quite often, the most predictable treatment possible is simply extraction. Because restoring the area with a dental implant or a conventional fixed prosthesis is more predictable. On the other hand, accomplishment of studies in this issue is challenging. Because it is difficult to find many teeth with similar vertical root fracture to evaluate the outcome of different treatment approaches. In the present patient, the lesion diagnosed as a vertical root fracture because the 
crack began from the root surface. Subadjacent to CEJ, presence of an inflammatory lesion on the gingival at the same area, suggested that the crack had extension into the root canal and probably, propagation of necrotic material through this crack resulted in occurrence of the gingival lesion. This crack was probably occurred as a result of combined effect of thin root wall pertain to internal root resorption and occlusal overload due to super contact.

Internal root resorption is rare in permanent teeth and is characterized by an oval-shaped enlargement of the root canal space (Glass and Zander, 1949). It is occurs due to resorption of the internal aspect of the root by multinucleated giant cells adjacent to granulation tissue in the pulp. Chronic inflammatory tissue is common in the pulp but only rarely it results in resorption. In addition to the requirement of the presence of granulation tissue, root resorption take place only if the odontoblastic layar and predentin are lost or altered (Inokoshi and Iwaku, 1982). Reasons for the loss of predentin adjacent to the granulation tissue are not obvious.

Trauma frequently has been suggested as a cause (Faraco and Holland, 2001). Some report that trauma may be recognized as an initiating factor in internal root resorption (Aeinehchi et al., 2003). Another reason for the loss of predentin might be extreme heat produced when cutting on dentin without an adequate water spray (Aeinehchi et al., 2003). Internal root resorption is usually asymptomatic and is first recognized clinically through routine radiographs. For internal resorption to be active at least part of the pulp, must be vital so that a positive response to pulp sensitivity testing is feasible. The coronal portion of the pulp is often necrotic whereas, the apical part which is includes the internal resorptive defect can remain vital. Therefore, a negative sensitivity test result does not rule out active internal resorption. It is also possible that the pulp becomes nonvital after a period of active resorption, giving a negative sensitivity test, radiographic signs of internal root resorption and radiographic signs of apical inflammation.

The patient did not remember any history of trauma to the mentioned tooth. So can orthodontic treatment lead to internal root resorption. Based on endodontics literature, occurrence of internal root resorption following the orthodontic treatment is infrequent and there are only few case reports. Kinomoto et al. (2002) reported occurrence of internal root resorption in the maxillary central and lateral incisors following the orthodontic treatment.

On the other hand, there are very few studies pertain to successful treatment of teeth with vertical root fracture, Harris (2000) in a case report described treatment of a tooth with vertical root fracture by resin-ionomer restoration. The crack was detected during a routine root coverage procedure. A resin-ionomer was used to repair the crack. Then, the root coverage procedure was completed. He reported that 15 months follow up showed the results remained stable and the tooth treated remained asymptomatic. In this case report, it seems that occlusal adjustment had a key role. Because a supra contact in a tooth with internal root resorption and thin root walls can contribute to further extension of the crack.

\section{CONCLUSION}

Despite, achievement of successful outcome in this case, it is not possible to anticipate long term survival for this tooth because thin walls of the root may result in further extension of the crack. It is necessary to emphasize that it is not possible to extend, the result of this treatment to all patients with vertical root fracture and at the present, there is no predictable treatment for such teeth and extraction may be the only choice of treatment.

\section{REFERENCES}

Aeinehchi, M., B. Eslami, M. Ghanbariha and A.S. Saffar, 2003. Mineral Trioxide Aggregate (MTA) and calcium hydroxide as pulp-capping agents in human teeth: $\mathrm{A}$ preliminary report. Int. Endod. J., 36: 225-231.

Andreasen, F.M., J. Daugaard-Jensen and E.C. Munksgaard, 1991. Reinforcement of bonded crown fractured incisors with porcelain veneers. Dent. Traumatol., 7: 78-83.

Bender, I.B. and J.B. Freedland, 1983a. Adult root fracture. J. Am. Dent. Assoc., 107: 413-419.

Bender, I.B. and J.B. Freedland, 1983b. Clinical considerations in the diagnosis and treatment of intra-alveolar root fractures. J. Am. Dent. Assoc., 107: 595-600.

Blaser, B.K., M.R. Lund, M.A. Cochran and R.H. Potter, 1983. Effects of designs of class 2 preparations on resistance of teeth to fracture. Oper. Dent., 8: 6-10.

Brown, W.S., H.R. Jacobs and R.E. Thompson, 1972. Thermal fatigue in teeth. J. Dent. Res., 51: 461-467.

Burke, F.J.T., 1992. Tooth fracture in vivo and in vitro. J. Dent., 20: 131-139.

Cameron, C.E., 1964. Cracked-tooth syndrome. J. Am. Dent. Assoc., 68: 405-411.

Cameron, C.E., 1976. The cracked tooth syndrome: Additional findings. J. Am. Dent. Assoc., 93: 971-975.

Carter, J.M., S.E. Sorensen, R.R. Johnson, R.L. Teitelbaum and M.S. Levine, 1983. Punch shear testing of extracted vital and endodontically treated teeth. J. Biomech., 16: 841-848. 
Caufield, J.B., 1981. Hairline tooth fracture: A clinical case report. J. Am. Dent. Assoc., 102: 501-502.

Cavel, W.T., W.P. Kelsey and R.J. Blankenau, 1985. An in vivo study of cuspal fracture. J. Prosthet. Dent., 53: 38-42.

Christensen, G.J., 1993. The cracked tooth syndrome: A pragmatic treatment approach. J. Am. Dent. Assoc., 124: 107-108.

Dang, D.A. and R.E. Walton, 1989. Vertical root fracture and root distortion: Effect of spreader design. J. Endodont., 15: 294-301.

Eakle, W.S., E.H. Maxwell and B.V. Braly, 1986. Fractures of posterior teeth in adults. J. Am. Dent. Assoc., 112: $215-218$.

Ehrmann, E.H. and M.J. Tyas, 1990. Cracked tooth syndrome: Diagnosis, treatment and correlation between symptoms and post-extraction findings. Aust. Dent. J., 35: 105-112.

Faraco Jr I.M. and R. Holland, 2001. Response of the pulp of dogs to capping with mineral trioxide aggregate or a calcium hydroxide cement. Dent Traumatol., 17: 163-166.

Feiglin, B., 1995. Clinical management of transverse root fractures. Dent. Clin. North Am., 39: 53-78.

Gher, Jr. M.E., R.M. Dunlap, M.H. Anderson and L.V. Kuhl, 1987. Clinical survey of fractured teeth. J. Am. Dent. Assoc., 114: 174-177.

Gibbs, J.W., 1954. Cuspal fracture odontalgia. Dent. Dig., 60: $158-160$.

Glass, R.L. and H.A. Zander, 1949. Pulp healing. J. Dent. Res., 28: 97-107.

Gutmann, J.L. and H. Rakusin, 1994. Endodontic and restorative management of incompletely fractured molar teeth. Int. Endod. J., 27: 343-348.

Gutmann, J.L. and J.W. Harrison, 1991. Surgical Endodontics. Medico Dental Media International, Inc., St. Louis, Missouri, ISBN-13: 9781891949104 , pp: 468.

Gutmann, J.L. and M.S. Gutmann, 1995. Cause, incidence and prevention of trauma to teeth. Dent. Clin. North Am., 39: 1-13.
Harper, R.H., R.J. Schnell, M.L. Swartz and R.W. Phillips, 1980. In vivo measurements of thermal diffusion through restorations of various materials. J. Prosthetic Dent., 43: 180-185.

Harrington, G.W., 1979. The perio-endo question: Differential diagnosis. Dent. Clin. North Am., 23: 673-690.

Harris, R.J., 2000. Treatment of a cracked tooth with a resin-ionomer restoration and a connective tissue graft: A case report. Int. J. Periodontics Restorative Dent., 20: 613-617.

Harvey, T.E., J.T. White and I.J. Leeb, 1981. Lateral condensation stress in root canals. J. Endodont, 7: $151-155$.

Hiatt, W.H., 1973. Incomplete crown-root fracture in pulpal-periodontal disease. J. Periodontol., 44: 369-379.

Holcomb, J.Q., D.L. Pitts and J.I. Nicholls, 1987. Further investigation of spreader loads required to cause vertical root fracture during lateral condensation. J. Endodont., 13: 277-284.

Howe, C.A. and D.J. McKendry, 1990. Effect of endodontic access preparation on resistance to crown-root fracture. J. Am. Dent. Assoc., 121: $712-715$.

Huang, T.J.G., H. Schilder andD. Nathanson, 1992. Effects of moisture content and endodontic treatment on some mechanical properties of human dentin. J. Endodont., 18: 209-215.

Inokoshi, S. and M. Iwaku, 1982. Pulpal response to a new adhesive resin material. J. Dent. Res., 61: 1014-1019.

Johnson, J.K., N.L. Schwartz and R.T. Blackwell, 1976. Evaluation and restoration of endodontically treated posterior teeth. J. Am. Dent. Assoc., 93: 597-605.

Josell, S.D., 1995. Evaluation, diagnosis and treatment of the traumatized patient. Dent. Clin. North Am., 39: 15-24.

Kinomoto, Y., T. Noro and S. Ebisu, 2002. Internal root resorption associated with inadequate caries removal and orthodontic therapy. J. Endod., 28: 405-407. 